La Charte d'Ottawa visait déjà à encourager les individus à exploiter tout leur potentiel en matière de santé. Avec la stratégie "Santé2020", le Conseil fédéral souhaite renforcer les compétences de la population dans ce domaine et permettre aux personnes atteintes de maladies chroniques à gagner en autonomie. Le programme Evivo est un parfait exemple en ce sens.

Dr med. Christine Romann, membre du Comité central de la FMH, responsable du département Promotion de la santé et prévention

\title{
E comme Evivo
}

\section{Michael Deppeler}

Dr med., spécialiste en médecine générale, médecin de famille, directeur médical et propriétaire de Salutomed AG

«Evivo - devenir acteur de sa santé» est un programme d'autogestion destiné aux personnes souffrant de maladies chroniques. Il a été mis au point il y a plus de 20 ans par Kate Lorig au Stanford Patient Education Center. Evivo s'intègre parfaitement au nouveau "credo" de Santé2020. Si les patients restent tributaires du "savoir-faire des professionnels», ils doivent être intégrés au processus de traitement de manière participative. Le programme a été mis en place en 2012 en Suisse romande et alémanique, ainsi que dans les pays européens de langue allemande.

Seuls, nous sommes des mots; réunis, nous formons un poème. (Georg Bydlinski)

Il y a 20 ans, mon prédécesseur reconnaissait à regret que l'âge d'or du médecin de famille était révolu. Depuis, la situation n'a fait qu'empirer. Les médecins de famille et les soins de base sont en passe de devenir un luxe. Il est soudain question de réseaux de soins, dernières forteresses contre la perte des privilèges. A l'époque, c'étaient les idées plus que les moyens financiers qui avaient fait défaut. Le système de santé est le reflet de notre société de l'information et de la mondialisation [1]*. Les mondes virtuels menacent-ils notre réalité? Le monde traditionnel peut-il être complété, voire perfectionné? Une nouvelle réflexion par le dialogue permettra-t-elle peut-être de résoudre des questions encore sans réponse? Le savoir se démocratise. Et les patients? Sont-ils plutôt au centre du pouvoir ou au bord de l'oubli?

"Pour faire évoluer le système de santé, il faut encore davantage intégrer la perspective des patients: leurs besoins doivent être au centre et les prestations s'axer plus fortement sur la prévention, les soins de longue durée des personnes souffrant de maladies chroniques et la fin de vie. Il s'agit également de développer leurs compétences en matière d'autogestion afin de les utiliser pour la prise en charge dans l'environnement privé.» [2]

La stratégie «Santé2020» du Conseil fédéral accorde une place plus importante aux patients. Mais leur donne-telle une voix? La Charte d'Ottawa [3] appelait déjà à la participation des patients. Où est passé ce pouvoir? $\mathrm{Ne}$ sommes-nous pas sous le joug de l'argent et de l'économie? Même les médecins sont considérés comme des troubles-fêtes au sein du système [4] ou des acteurs à adapter au «modèle d'affaires» qu'est devenue la santé [5].

\section{Santé: fruit de l'éducation et de la relation}

Une nouvelle solidarité doit se faire jour au sein du système de santé. La relation avec le patient et les proches en constitue la clé de voûte. Sans relation, pas de coopération - et encore moins d'autogestion. Tous les patients se "gèrent» eux-mêmes, ils ont appris à le faire au cours de leur "carrière de patient», tout particulièrement les patients polymorbides. Si la génétique est une racine, l'environnement social devient le terreau de la santé et l'éducation en est l'engrais. dialog-gesundheit [6] nous apprend que la perception au sein de la population peut être très différente de celle des professionnels (et du monde politique). Trop souvent, les solutions descendantes (projets) divergent des besoins ascendants (processus) [7]. Les gens souhaitent prendre leur vie en main. Leur autonomie commence par l'écoute (active) et par le fait de (pouvoir) raconter son ou ses histoires. A la clé: compréhension, confiance et responsabilité.

L'enquête européenne sur les compétences en matière de santé l'a montré: les patients souffrant de maladies chroniques ont souvent un niveau de compétences faible ou insuffisant en matière de santé [8]. Le Chronic Disease Self-Management Program (CDSMP) se présente comme l'une des solutions les plus prometteuses à ce sujet. Perfectionnée depuis plus de 30 ans au Stanford Patient Education Center [9], elle est mise à la disposition de différents groupes cibles dans plusieurs configurations possibles 
[10]. Il y a trois ans, la Fondation Careum [11] a adapté ce programme pour la Suisse alémanique et la Suisse francophone; il est intitulé «Evivo - devenir acteur de sa santé». Evivo est transpathologique et part du principe que les personnes atteintes de maladies chroniques ont des soucis communs et des défis similaires à relever [12]. Elles ont toutes le droit de participer, ainsi que les proches aidants.

«Ma femme (79 ans) et moi-même (81 ans) avons participé en tant que patiente et proche aidant. Le plus important était le plan d'action: ma femme ne pouvait plus jouer de violon. Elle a donc opté pour la flutte pour retrouver une occasion de faire de la musique. Pendant plusieurs dizaines d'années, j'avais moi-même effectuéle parcours Vita plusieurs fois par semaine. Avec la réorganisation du ménage, j'ai pu reprendre mes sorties. Je suis ancien médecin de famille et le cours m'a tellement impressionné que j'ai accepté d'aider à informer et à faire la publicité du programme." (D. Künzler, participant, ancien médecin de famille)

Le programme Evivo dure 6 semaines. Les responsables de groupe suivent une formation et disposent d'un guide qui les aide à respecter le déroulement structuré des unités de cours hebdomadaires de deux heures et demie. La plupart d'entre eux sont d'anciens participants ayant eux-mêmes une expérience face à la maladie. Les sujets abordés sont entre autres: alimentation, exercice physique, douleur, anxiété, fatigue, prise de décision ou encore gestion des médicaments. Le manuel personnel faisant office de support de cours comporte des informations sur la santé [13].

"Evivo est très utile pour aider à venir à bout du quotidien en dépit de la maladie chronique; le programme encourage la prise en charge du patient par lui-même. J'ai été reconnaissante de voir comment les participants retrouvaient goût à la vie, comme ils envisageaient ensuite leur vie avec plus de confiance et d'assurance. A titre personnel, je pense que ce cours est un complément précieux et utile en soutien du travail accompli par les médecins de famille et le personnel soignant.» (E. Schaad, responsable de groupe et participante)

Plus de 500 personnes ont participé à 49 cours. 115 responsables de groupes et 9 coaches ont été formés. L'association «Réseau Evivo» a été créée par onze organisations à l'été 2014 à Zurich. Les réactions des patients sont excellentes. Ils apprécient particulièrement le plan d'action et l'élaboration d'objectifs concrets qui restent à leur portée. Ces outils permettent d'amorcer des changements de comportement, un surplus de motivation et un sentiment d'efficacité personnelle, tout en esquissant des pistes pour rompre l'isolement social.

"Pour le dire de façon simple, l'autogestion consiste à mettre en cuvre des stratégies personnelles pour vivre sa vie selon ses attentes, ses idéaux et ses besoins, en dépit des défis que pose la maladie chroniqueque l'on soit touché directement ou indirectement.» (K. Lorig [13])

\section{Les soins de base au centre}

Dr Michael Deppeler

Salutomed AG

Kirchlindachstrasse 7

CH-3052 Zollikofen

m.deppeler[at]salutomed.ch

Les mesures de marketing classiques sont trop imper- sonnelles et se noient dans la masse publicitaire. Les relations de longue date que nous entretenons avec les patients nous confèrent un rôle et une place particulière à leurs yeux. Nous pourrions aider à ancrer Evivo dans les structures de soins des réseaux régionaux, à l'image de ce qui se fait avec le coaching santé. Une sorte de nouvelle coopération entre médecin, pharmacien et soignants incluant une formation des adultes pourrait voir le jour avec les objectifs suivants: amélioration de la qualité de vie, succès cliniques, utilisation efficace des prestations de soins, renforcement de la confiance en soi, effets positifs sur les relations interpersonnelles dans l'environnement social, encouragement des performances chez les personnes actives ou incitation à l'engagement bénévole [14]. Le succès des cours Evivo profitera à tous. Dans notre quotidien, le retour sur investissement, souvent évoqué, se ressent et libère de nouvelles ressources, "réinvesties» dans l'«empowerment» de patients disposant de compétences moindres en matière de santé. Notre approche, à savoir mener des entretiens axés sur la motivation, vise à agir sur les comportements et à renforcer le sentiment d'efficacité personnelle [15].

\section{Si l'enseignement vient d'experts, les patients en savent plus; en revanche, ils agissent davantage si les conseils sont donnés par des personnes qui ont été concernées.}

L'approche "peer-to-peer» fournit une base essentielle. Le soutien social est indispensable à la promotion de l'autogestion [16]. Du point de vue du patient, les «peers» sont vécus comme un tournant dans l'évolution de la maladie. Dès lors, il leur est possible d'adopter une perspective différente sur l'avenir. Cette pertinence croissante du soutien "peer-to-peer» se reflète sur Internet et les réseaux sociaux. En tant que professionnels, nous restons la première source d'information pour les malades chroniques [17]. Plus le système de santé devient complexe, plus les personnes de confiance gagnent en importance dans différents rôles: en tant qu'experts du "dialogue», du "coaching» et du "leadership» dans le domaine de la santé publique et de la prévention. En tant qu' "experts du savoir», nous ne sommes plus seuls. Les malades chroniques sont, quant à eux, des «experts de l'expérience» et font, à ce titre, partie intégrante des soins de base du futur [18]. Dans le même temps, les approches "peer» soulèvent de nouveaux défis. L'expérience vécue de la maladie peut se révéler problématique quand, du point de vue du malade, elle revient à anticiper sa propre souffrance [19]. Cela suppose une solide formation et un suivi constant des "peers» est-ce une mission supplémentaire dévolue aux nouveaux «soins de base»?

La plus belle récompense de nos efforts n'est pas ce que nous recevons en retour, mais ce que nous devenons à travers eux. (John Ruskin) 


\section{Références}

1 Cycle de Kondratiev (Wikipédia).

2 Département Fédéral de l'Intérieur (DFI). Santé2020. Politique de la santé: les priorités du Conseil fédéral. Berne: Office fédéral de la santé publique; 2013

3 OMS Organisation Mondiale de la Santé. Charte d'Ottawa pour la promotion de la santé. Genève; 1986.

4 Unschuld PU. Ware Gesundheit - Das Ende der Klassischen Medizin. C.H. Beck

5 Maio G. Geschäftsmodell Gesundheit.dialog-gesundheit (www. dialog-gesundheit.ch)

7 Syme LS. Community Health Berkeley, nationale Gesundheitsförderungskonferenz. Freiburg; 2005

8 Sørensen, K, Pelikan JM, Röthlin F, Ganahl K, Slonska Z, Doyle, Brand $\mathrm{H}$. Health literacy in Europe: comparative results of the European health literacy survey (HLS-EU). European Journal of Public Health. 2015. doi:10.1093/eurpub/ckv043

9 Lorig K R, Ritter P, Stewart AL, Sobel DS, Brown BW, Bandura BWet al. Chronic disease self-management program: 2-year health status and health care utilization outcomes. Medical Care. 2001; 39(11):1217-23.

10 Brady TJ, Murphy L, O'Colmain BJ, Beauchesne D, Daniels B, Greenberg, et al. A meta-analysis of health status, health behaviors, and healthcare utilization outcomes of the Chronic Disease Self-Management Program. Preventing Chronic Disease. 2013; 10.120112. doi:10.5888/pcd10.120112

11 Haslbeck J. Selbstmanagementförderung. Empowerment zu gesundheitsbewusstem Leben mit chronischer Krankheit am Beispiel von Evivo. Care Management. 2012;5(1)23-6.
12 Schaeffer, D., \& Haslbeck, J. (2015). Bewältigung chronischer Krankheit. In Soziologie von Gesundheit und Krankheit. Ein Lehrbuch (p. in print). Wiesbaden: Springer VS Verlag.

13 Lorig KR, Holman H, Sobel DS, Laurent D, Gonzales VM, Minor M. Vivre sainement et activement avec une maladie chronique. Zurich: Careum Verlag. 2013

14 Expert Patients Programme. Healthy lives equal healthy communities - the social impact of self-management. London: EPP CIC; 2011.

15 De Silva D. Evidence: Helping people help themselves. A review of the evidence considering whether it is worthwhile to support self-management. London: The Health Foundation; 2011.

16 Taylor S J, Pinnock H, Epiphaniou E, Pearce G, Parke HL, Schwappach $\mathrm{A}$, et al. A rapid synthesis of the evidence on interventions supporting self-management for people with long-term conditions: PRISMS - Practical systematic Review of Self-Management Support for long-term conditions. Health Services and Delivery Research. 2014;2(53):1-580. doi:10.3310/hsdr02530

17 Marstedt G. Gesundheitsfragen - Information und Wissen de Bürger. In Böcken J, Braun B, Landmann J (Eds.). Gesundheitsmonitor 2010. Bürgerorientierung im Gesundheitswesen (S. 43-89). Gütersloh: Verlag Bertelsmann Stiftung; 2010.

18 Kämpfer T, Schwager C. Betroffene beraten Betroffene. Wie am Schweizer Paraplegiker-Zentrum Nottwil Patienten geschult werden. Care Management. 20092(2):24-7.

19 Schaeffer D. Der Patient als Nutzer. Bern: Hans Huber; 2004. 